\title{
Maintenance of spermatogenesis requires TAF4b, a gonad-specific subunit of TFIID
}

\author{
Allison E. Falender, ${ }^{1}$ Richard N. Freiman, ${ }^{3}$ Kenneth G. Geles, ${ }^{4}$ Kirk C. Lo, ${ }^{2,5}$ KeumSil Hwang, ${ }^{6}$ \\ Dolores J. Lamb, ${ }^{1,2}$ Patricia L. Morris, ${ }^{6}$ Robert Tjian, ${ }^{4}$ and JoAnne S. Richards ${ }^{1,7}$ \\ ${ }^{1}$ Department of Molecular and Cellular Biology, ${ }^{2}$ Scott Department of Urology, Baylor College of Medicine, Houston, Texas \\ 77030, USA; ${ }^{3}$ Department of Molecular and Cell Biology and Biochemistry, Brown University, Providence, Rhode Island \\ 02921, USA; ${ }^{4}$ Howard Hughes Medical Institute, Department of Molecular and Cell Biology, University of California at \\ Berkeley, Berkeley, California 94720, USA; ${ }^{5}$ Mount Sinai Hospital, Division of Urology, University of Toronto, Toronto, \\ Ontario, Canada M5G 1X5; ${ }^{6}$ Population Council, The Rockefeller University, New York, New York 10021, USA
}

\begin{abstract}
The establishment and maintenance of spermatogenesis in mammals requires specialized networks of gene expression programs in the testis. The gonad-specific TAF4b component of TFIID (formerly TAF 105 ) is a transcriptional regulator enriched in the mouse testis. Herein we show that TAF4b is required for maintenance of spermatogenesis in the mouse. While young Taf $4 b$-null males are initially fertile, Taf $4 b$-null males become infertile by 3 mo of age and eventually exhibit seminiferous tubules devoid of germ cells. At birth, testes of $T a f 4 b$-null males appear histologically normal; however, at post-natal day 3 gonocyte proliferation is impaired and expression of spermatogonial stem cell markers c-Ret, Plzf, and Stra8 is reduced. Together, these data indicate that TAF4b is required for the precise expression of gene products essential for germ cell proliferation and suggest that TAF4b may be required for the regulation of spermatogonial stem cell specification and proliferation that is obligatory for normal spermatogenic maintenance in the adult.
\end{abstract}

[Keywords: Spermatogonial stem cell; Taf4b; germ cell; Stra8; c-Ret]

Supplemental material is available at http://www.genesdev.org.

Received December 15, 2004; revised version accepted February 17, 2005.

Spermatogenesis is a complex process requiring the specialized function of multiple cell types including somatic and germ cells that collectively results in the continuous production of functional sperm in adult males. The unlimited production of male gametes is largely accomplished through the ability of spermatogonial stem cells to self-renew in the adult testis. These complex and multifaceted events are dependent on appropriate expression and action of specific genes at multiple stages of germ cell and testicular development (Matzuk and Lamb 2002; McLaren 2003). The precise temporal and spatial expression of specific transcription factors is also essential for proper execution of spermatogenesis (SassoneCorsi 1997). Emerging evidence now suggests that in addition to gonad-specific transcription factors, specialized components of the basal RNA Polymerase II machinery are also critical for the execution of gonad-specific programs of gene expression (Hochheimer and Tjian 2003).

The TFIID complex is a core RNA polymerase complex that contains the TATA-binding protein (TBP) and

${ }^{7}$ Corresponding author.

E-MAIL joanner@bcm.tmc.edu; FAX (713) 790-1275.

Article published online ahead of print. Article and publication date are at http://www.genesdev.org/cgi/doi/10.1101/gad.1290105.
14 TBP-associated factors (TAFs) that function in core promoter recognition and activator-dependent RNA Polymerase II recruitment (Verrijzer and Tjian 1996). While most TFIID subunits are expressed and function broadly in most cell types, there are selective TFIID subunits that apparently have evolved to function in the specification of gonadal-specific programs of gene expression. In the mouse, TAF4b is a component of TFIID that is highly enriched in gonadal tissues and is required for ovarian follicle development (Freiman et al. 2001). TAF4b is similar in structure to its broadly expressed paralog TAF4 (TAF 1 II 13 ). While TAF4 and TAF4b display overlapping expression patterns in certain cell types, TAF4b is essential for regulating the selective expression of ovarian-specific gene expression patterns required for female fertility (Freiman et al. 2001).

Several other members of the basal transcription machinery also exhibit cell-specific expression and regulation of genes in the testis. The Drosophila testes contain several unique isoforms of TAFs that are essential for fertility (Hiller et al. 2004). In mammals, TFIID components are expressed at high levels at specific stages of spermatogenesis (Schmidt and Schibler 1995) and the testis contains a unique paralog of TAF7, TAF7L (Pointud et al. 2003). TRF2/TLF, a TBP-related factor, acts as 
a surrogate transcription factor for TBP in pachytene spermatocytes and post-meiotic spermatids (Martianov et al. 2002). Loss of Trf2/Tlf causes infertility in mice (Zhang et al. 2001). ALF (TFIIA $\tau$ ) is a germ cell-specific isoform of TFIIA, another component of the basal RNA Polymerase II machinery, that can substitute for TFIIA at specific promoters (Ozer et al. 2000). The requirement for specialized transcription machinery in spermatogenesis is a highly conserved phenomenon across species and likely involves several yet-to-be-characterized factors.

To identify the function of TAF4b in male fertility, we characterized reproduction and testis development in the Taf4b-null males. Herein we show that TAF4b is obligatory for the maintenance of spermatogenesis in adult mice. Mice null for Taf $4 b$ initially appear normal and fertile; however, following the first round of spermatogenesis, seminiferous tubules degenerate, eventually becoming devoid of germ cells. Phenotypic abnormalities are first apparent at post-natal day 2 when germ cell stem cell differentiation and proliferation are disrupted and several genes involved in spermatogonial stem cell function including Stra8 and $c$-Ret are misregulated. These data suggest that early expression of TAF4b in spermatogonial stem cells in the post-natal testis may be required for the proper maintenance of spermatogenesis in the adult.

\section{Results}

Taf4b-null males become infertile by 11 wk of age

Taf $4 b$-null males are initially fertile, but display multiple reproductive defects by $11 \mathrm{wk}$ of age. To investigate the underlying cause for loss of fertility, 4-wk-old Taf $4 b$-null or heterozygous male mice were placed with fertile females. The experiment began on the day that the first litter was born, and the number of subsequent litters born was recorded for 6 mo. During the first month, Taf $4 b$ heterozygous and null males sired a comparable number of litters (Fig. 1A). When the number of pups born to each female was counted at the time of weaning, Taf $4 b$ heterozygotes and null mice sired a similar number of pups (Fig. 1B). During months 2-6, Taf $4 b$ heterozygotes sired an average of four litters. In contrast, no litters were born to Taf $4 b$-null males after the first month of mating (Fig. 1A).

To determine more precisely when $T a f 4 b$-null males become infertile, immature wild-type females were treated with a superovulatory regime of hormones and placed in cages with a single 8 -wk-old Taf $4 b$ heterozygous or null male. On the following morning, vaginal plugs were observed, and on the next day females were sacrificed, oviducts were removed, and the number of fertilized versus nonfertilized oocytes was counted. In superovulated females placed with either 8-wk-old Taf $4 b$ heterozygotes or knockouts, $71 \%$ and $59 \%$ of oocytes were fertilized, respectively (Fig. 1C). When the experiment was repeated with the same males but new females at $11 \mathrm{wk}$ of age, $78 \%$ of the oocytes were fertil-

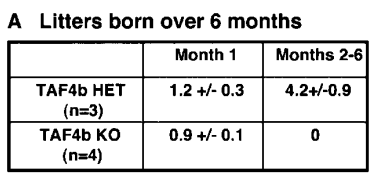

B Average size of first litter

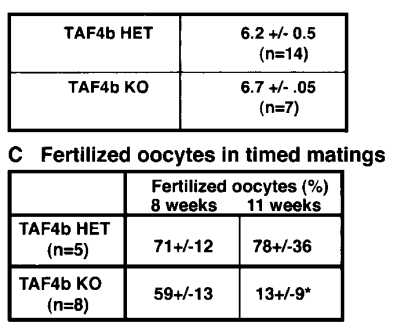

G Measures of sperm function

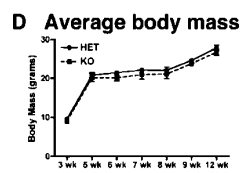

E Average testis mass
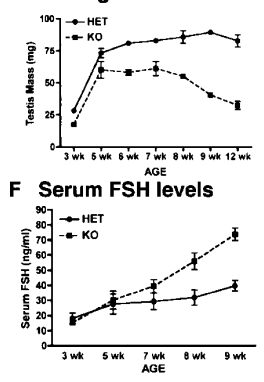

\begin{tabular}{|l|c|c|c|c|c|c|}
\hline & \multicolumn{2}{|c|}{$\begin{array}{c}\text { Sperm Count } \\
\left(\times 10^{\wedge} 6 / \mathrm{ml}\right)\end{array}$} & \multicolumn{2}{c|}{$\begin{array}{c}\text { Sperm Motility } \\
(\%)\end{array}$} & \multicolumn{2}{c|}{$\begin{array}{c}\text { Acrosome Cap } \\
(\%)\end{array}$} \\
& 8 weeks 12 weeks & 8 weeks 12 weeks & 8 weeks 12 weeks \\
\hline TAF4b HET & $19.3+/-4.2$ & $24.7+/-7.2$ & $39+/-6.8$ & $36+/-11.9$ & $86+/-3.9$ & $95+/-1.7$ \\
\hline TAF4b KO & $12.0+/-4.0^{*}$ & $2.6+/-2.2^{*}$ & $33+/-6.8$ & $7+/-3.0^{*}$ & $75+/-5.2^{*}$ & $46+/-7.9^{*}$ \\
\hline
\end{tabular}

Figure 1. $(A)$ The number of litters born during the first month or months 2-6 from breeding pairs with Taf $4 b$ heterozygous or null males were counted. $(B)$ The average number of pups born in the first litter to Taf $4 b$ heterozygous or null males was counted. $(C)$ The percentage of oocytes fertilized in superovulated wild-type females placed with $T a f 4 b$ heterozygous or null males at 8 and 11 wk of age was determined. Means were calculated (as shown by a solid bar), and statistical significance was determined using the Student's $t$-test. $(D)$ Taf $4 b$ heterozygous and null males were weighed. $(E)$ Testes weights from the same mice were weighed. Means were compared by two-way ANOVA. An asterisk $\left({ }^{\star}\right)$ indicates $p<0.01$. $(F)$ Serum from Taf $4 b$ heterozygous and null males was isolated and analyzed by radioimmunoassay for FSH. Statistical analysis was performed using two-way ANOVA. Statistical significances of $p<0.01\left(^{\star}\right)$ and $p<0.001\left(^{\star \star}\right)$ are indicated.

ized by $T a f 4 b$ heterozygotes. In contrast, only $13 \%$ of the oocytes collected from superovulated females placed with 11-wk-old Taf4b-null males were fertilized (Fig. 1C). Therefore, Taf $4 b$ heterozygotes and knockouts are able to fertilize oocytes in vivo at comparable levels when they are $8 \mathrm{wk}$ of age but 11 -wk-old Taf $4 b$-null males are essentially infertile.

To assess testes development, body weight and testes weights were measured. At all time points there was no significant difference in the body mass of Taf $4 b$-null and heterozygous mice (Fig. 1D). However, there was a significant difference in testis weights between Taf $4 b$ heterozygotes and knockouts as early as 3 wk of age $(p<0.01)$ (Fig. 1E). By 6 wk of age, Taf $4 b$-heterozygous testes reached near maximal mass, weighing $81.1 \mathrm{mg}$ $( \pm 1.4)$ on average, that was maintained through $12 \mathrm{wk}$. In contrast, Taf $4 b$-null testes were smaller at 6 wk of age $(58.1 \mathrm{mg} \pm 2.2 \mathrm{mg})$ and weighed only $40 \%$ that of heterozygotes at $12 \mathrm{wk}$.

To determine if endocrine abnormalities might underlie or contribute to the Taf $4 b$ male phenotype, serum levels of follicle stimulating hormone (FSH), luteinizing hormone ( $\mathrm{LH})$, and testosterone $(\mathrm{T})$ were measured. In mice younger than 7 wk of age, no differences were ob- 
served in serum levels of FSH, LH, or T (Fig. 1F; data not shown). However, by 8-9 wk of age, FSH levels become elevated in Taf $4 b$-null males relative to heterozygous littermates. Serum levels of $\mathrm{LH}$ and $\mathrm{T}$ remained comparable in the heterozygous and null animals at all ages assayed (data not shown). Fertile 8-wk-old Taf4b-null males exhibited minor defects in epididymal sperm; however, by 12 wk sperm motility and acrosome formation were severely impaired (Fig. 1G).

TAF4B is expressed in gonocytes in the post-natal testes and in spermatogonia and spermatids in the adult testes

To determine the cell type or stage-specific expression of TAF4B, immunohistochemistry using a polyclonal TAF4B antiserum was performed. The results demonstrate that TAF4b protein is expressed highly in gonocytes in neonatal testes (Fig. 2A, day 3). In the adult testes (Fig. 2B) specific punctate nuclear staining was observed in premeiotic spermatogonia and post-meiotic spermatids. TAF4b protein was not detected in meiotic spermatocytes or somatic cells. TAF4b protein was not detected in Taf $4 b$-null testes (Fig. 2C) or in wild-type testes incubated with preimmune serum (Fig. 2D). Immunohistochemical analysis in wild-type adult testes ranging in age from $12 \mathrm{wk}$ to $1 \mathrm{yr}$ showed that TAF4b protein was detected in the nuclei of spermatogonia and spermatids but not other testis cell types at all ages assayed (data not shown). A Western blot using the same antibody produced a $105-\mathrm{kDA}$ band in adult heterozygous testes but not Taf $4 b$-null testes (Fig. 2E). Thus, TAF4b protein is expressed in germ cells of neonatal as well as adult mice, suggesting that TAF4b might function at several stages of testes development and spermatogenesis.

\section{Taf4b-null testes degenerate as males age}

To determine if changes in germ cell number or maturation occur and underlie the defects in Taf4b-null testes, sections from heterozygous and null animals of various ages were stained with hemotoxylin and eosin or immunostained for germ cell nuclear antigen 1 (GCNA1) used as a marker for premeiotic and meiotic germ cells (Enders and May 1994). No overt histological differences were observed in neonatal (day 0) Taf $4 b$-null and heterozygous testes (Fig. 3A-D). However, fewer germ cells were present in Taf $4 b$-null testes at post-natal day 8 (Fig. $3 \mathrm{E}-\mathrm{H})$. In testes from day 21 , Taf $4 b$-null mice, the lumens of seminiferous tubules appear to be less densely packed compared to heterozygous males (Fig. 3I,K). GCNA1 staining of these same testes revealed that a few seminiferous tubules of Taf $4 b$-null mice were devoid of germ cells and the tubules that did contain sperm were less densely packed with cells relative to heterozygous littermates (Fig. 3J,L). By 8 wk of age, the seminiferous tubules of $T a f 4 b$ heterozygous and null mice contain

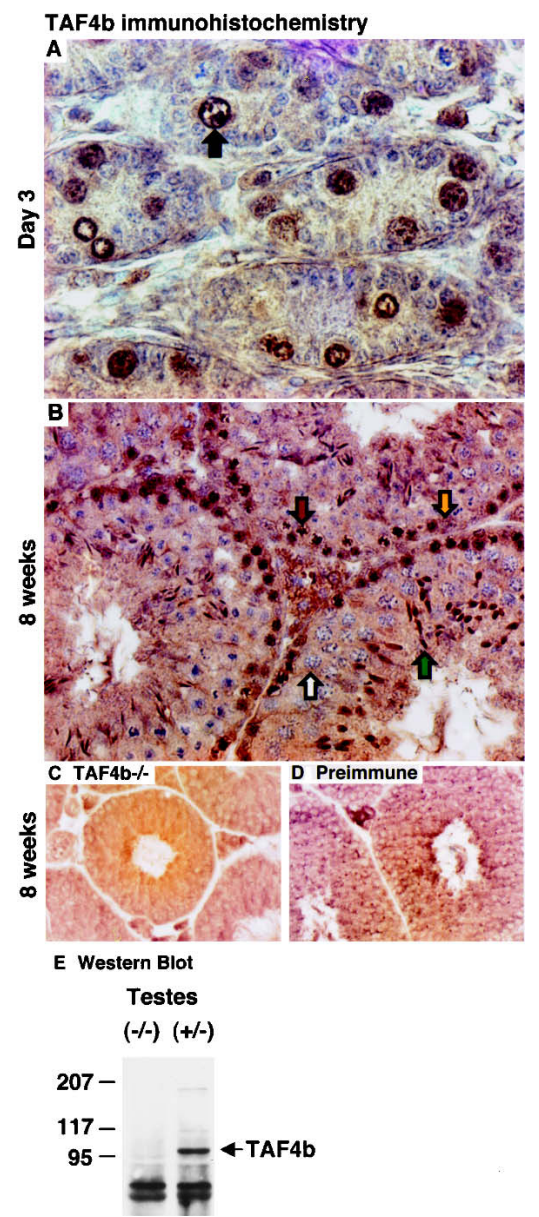

Figure 2. Testes from wild-type 3-d-old $(A)$ and 8 -wk-old $(B)$ or Taf $4 b$-null 8-wk-old $(C)$ animals were immunostained with antiserum against TAF4b. Preimmune serum was used to stain wild-type 8-wk-old testes $(D)$. The following cell types are indicated by arrows: gonocytes (black), spermatogonia (red), Sertoli cells (yellow), spermatocytes (white), and spermatids (green). (E) Western blots with whole-cell extracts from adult Taf $4 b$-null or heterozygous testes were probed with TAF4b antiserum.

maturing sperm (Fig. 3M,O). However, some Taf4b-null seminiferous tubules were conspicuously devoid of sperm. At 12 wk of age normal spermatogenesis was observed in all tubules of heterozygous mice (Fig. 3Q,R), whereas Taf $4 b$-null tubules contained reduced numbers of germ cells (Fig. 3S,T). By 8 mo seminiferous tubule degeneration including loss of germ cells was apparent even in Taf $4 b$ heterozygotes (Fig. $3 \mathrm{U}, \mathrm{V}$ ); however, heterozygous males remain fertile beyond 12 mo (data not shown). Conversely, by 8 mo Taf $4 b$-null males are not only infertile but also present seminiferous tubules that with few exceptions are completely devoid of sperm (Fig. $3 \mathrm{~W}, \mathrm{X})$. Leydig cell hyperplasia was also apparent but was not associated with abnormal levels of testosterone or LH and is therefore most likely secondary to germ cell loss (Fig. 3X). 


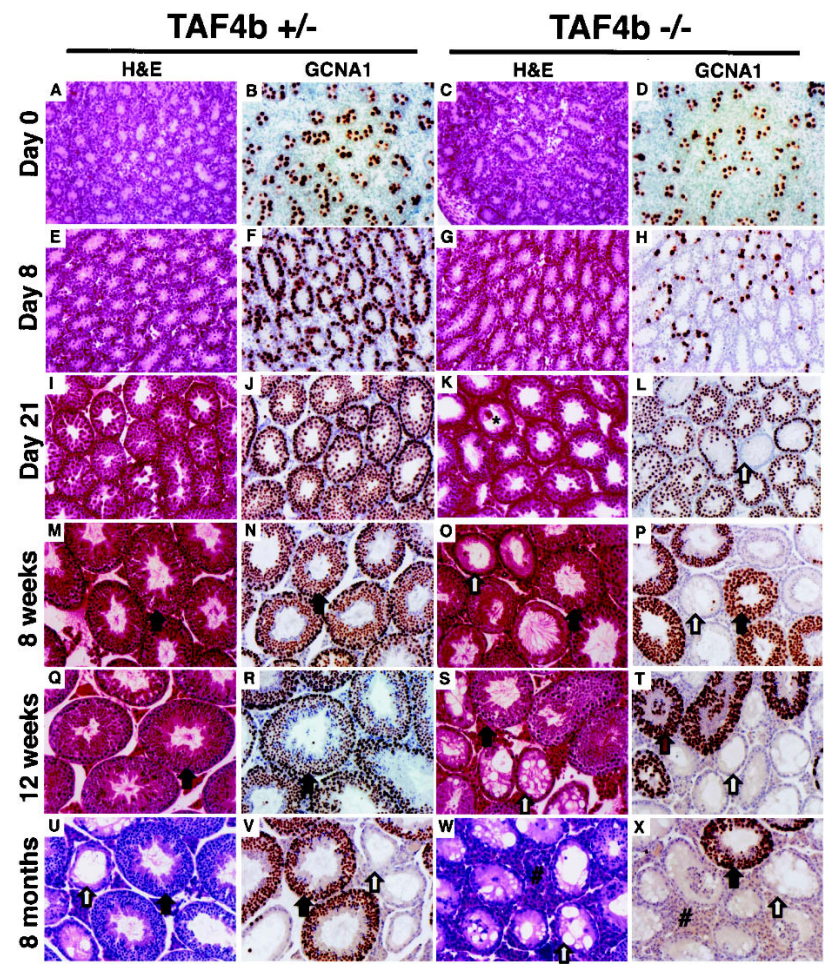

Figure 3. Testes from heterozygous (columns 1 and 2) and Taf $4 b$-null (columns 3 and 4) mice of various ages (day $8[A-D]$, day $21[E-H], 8 \mathrm{wk}[I-L], 12 \mathrm{wk}[M-P]$, and $8 \mathrm{mo}[Q-T])$ were stained with hematoxylin and eosin (columns 1 and 3) or immunostained with an antibody against GCNA1 (columns 2 and 4). Disrupted tubules in day 21 males are indicated by an asterisk $\left({ }^{\star}\right)$. Black arrows indicate normal seminiferous tubule morphology. Tubules depleted of germ cells are designated with open arrows. Red arrows indicate tubules in which germ cells are sloughing off. Leydig cell hyperplasia is marked with a pound sign $(\#)$.

\section{Germ cell proliferation is impaired in Taf4b-null males at post-natal day 2}

To investigate the underlying cause of the severe loss of germ cells in the adult Taf $4 b$-null males, gonads of neonatal mice were analyzed in greater detail. Immunostaining for GCNA1 revealed that on the day of birth (day 0) and on post-natal day 1, Taf $4 b$-null males have the same number of gonocytes per seminiferous tubule as wild-type and heterozygous testes (Fig. 4A). However, by post-natal day 2, wild-type and heterozygous mice have more gonocytes per seminiferous tubule than the Taf $4 b$-null mice. This difference persists through day 3 . By day 8 when there were only 3.8 spermatogonia in seminiferous tubules of Taf $4 b$-null mice, wild-type and heterozygous seminiferous tubules contained 14.4 and 14.9 spermatogonia per tubule, respectively (Fig. 4B).

In the mouse testes shortly after birth gonocytes migrate from the center of the lumen to the basement membrane of the seminiferous tubules, proliferate, and differentiate into spermatogonia. Functional spermatogonial stem cells are first evident at post-natal day 3 (McLean et al. 2003). Wild-type, heterozygous, and
Taf4b-null males were immunostained for phospho-histone H3 Ser 10, a marker of mitosis (Fig. 4D). At this important stage of germ cell proliferation, $45.4 \%$ and $47.6 \%$ of wild-type and heterozygous seminiferous tubules contain phospho-histone $\mathrm{H} 3$ positive cells, whereas only $26.6 \%$ of knockout seminiferous tubules contain positive cells $(p<0.05)$ (Fig. 4C). TUNEL staining to identify apoptotic cells failed to show differences in wild-type versus Taf $4 b$-null testes. Therefore, apoptosis is not the underlying cause of low germ cell number in Taf4b-null testes on day 3 or in the adult (Fig. 4E; data not shown).

\section{Loss of germ cells in Taf4b-null animals is cell autonomous}

To determine if loss of germ cells in Taf $4 b$-null males resulted from defective somatic cell support of germ cells or was inherent to germ cells, germ cell transplantation experiments were conducted. Germ cell stem cells isolated from Rosa26 mice were injected into the seminiferous tubules via the rete testes of 6-mo-old Taf4b-null testes. Germ cell-deficient $\mathrm{Kit}^{\mathrm{W}-\mathrm{V}} / \mathrm{Kit}^{W}$ mouse testes were also transplanted to serve as positive controls. Three months after the surgery, the animals were sacrificed and the testes were stained for $\beta$-galactosidase. Successful germ cell colonization was observed in multiple animals in both $\mathrm{Kit}^{W-v} / \mathrm{Kit}^{W}$ (Fig. 5A) and Taf4b-null (Fig. 5B) strains with donor germ cell derived spermatogenesis (Fig. 5E), suggesting that like the $\mathrm{Kit}^{W-v} / \mathrm{Kit}^{W}$ mice, the defects in Taf $4 b$-null mice are cellautonomous and inherent to the germ cells. In fact, the overall colonization success was superior in the Taf $4 b$ null mice with $>50 \%$ of the tubules staining blue. These data show that Taf4b-null Sertoli cells are functional and capable of forming proper cell contact with wild-type germ cell stem cells and maintaining spermatogenesis. No spermatogenesis was observed in nontransplanted testes (Fig. 5C,F).

Genes involved in germ cell stem cell function, retinoic acid signaling, meiosis, and spermatogenesis are misexpressed in post-natal Taf4b-null testes

Because germ cell stem cells are first present at day 3, germ cell stem cell markers were assayed by RT-PCR and microarray. As early as the day of birth, Stra8 (stimulated by retinoic acid 8), a germ cell stem cell marker (Giuili et al. 2002) is expressed at lower levels in Taf4bnull males (Fig. 6A). Stra8 expression remained lower in Taf $4 b$-null testes at day 8 (Fig. 6B) and in the adult (data not shown). Plzf, a transcriptional repressor required for germ cell stem cell self-renewal (Buaas et al. 2004), is expressed at normal levels in newborn Taf4b-null testes but becomes deficient by day 3 and remains low in day 8 Taf4b-null testes. GDNF (glial cell line-derived neurotrophic factor) is a factor secreted by Sertoli cells that mediates the decision between germ cell stem cell renewal and spermatogonial differentiation (Meng et al. 
Falender et al.

Figure 4. Wild-type, heterozygous, and Taf $4 b$-null testes were immunostained for GCNA1 at post-natal days $0,1,2,3(A)$, and $8(B)$, and the number of positive cells per seminiferous tubule was counted $l^{\star *}$ indicates $p<0.05)$. The same testes were stained for phospho-histone H3 (Ser 10) (D) or TUNEL-positive cells (E). Positive cells are indicated by black arrows. $(C)$ The percentage of tubules with phospho-histone H3 (Ser 10) positive cells was counted.
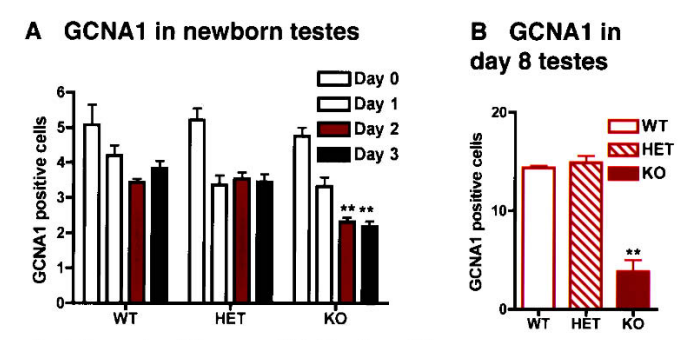

C Phospho-Histone H3 (Serine 10) in day 3 testes
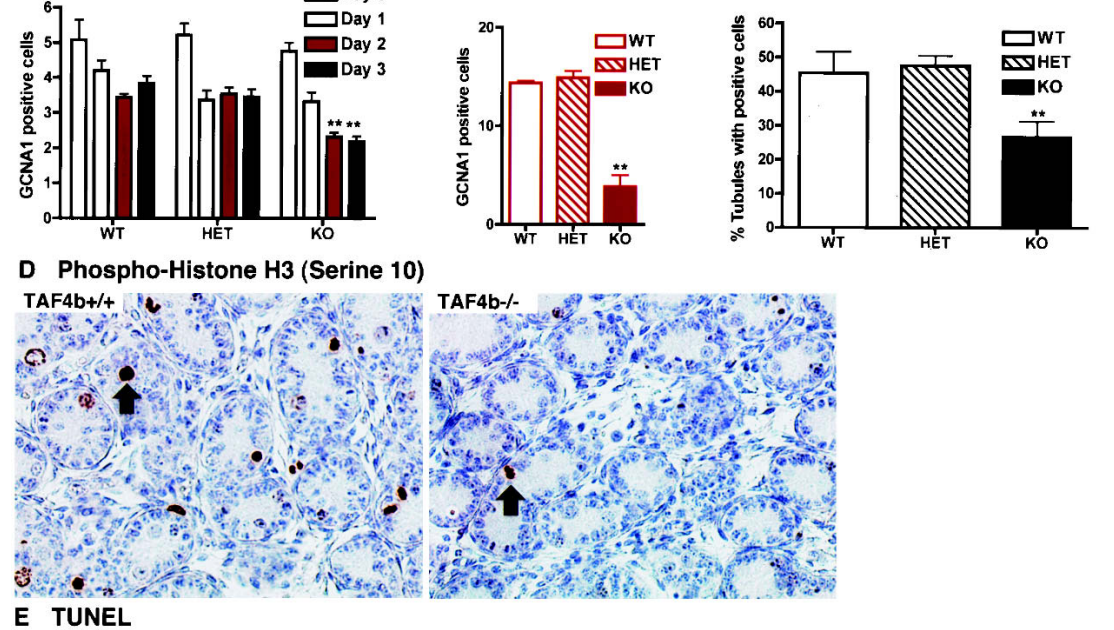

\section{E TUNEL}

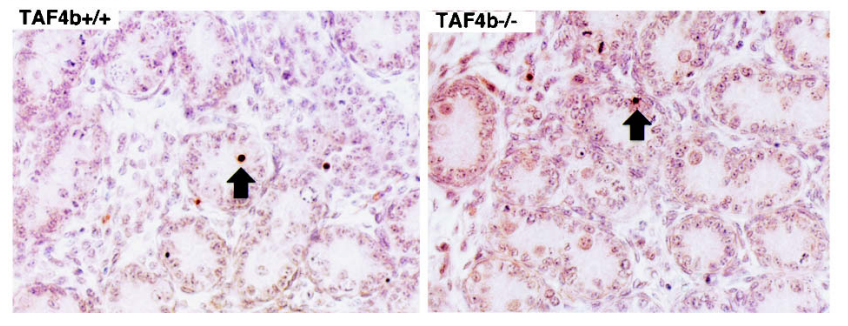

2000). Gdnf is expressed at normal levels in Taf4b-null males, however, the GDNF receptor Gfr1 $\alpha$ and its effector tyrosine kinase receptor $c$-Ret are deficient in day 8, Taf4b-null testes (Fig. 6A,B) with lower $c$-Ret expression observed as early as the day of birth. These results suggest that early post-natal germ cell stem cell specification and proliferation are impaired in Taf $4 b$-null males, likely resulting in fewer germ cell stem cells.

Several genes known to be important to meiosis and spermatogenesis are also expressed at reduced levels in the Taf $4 b$-null testis at post-natal day 8 , a time when the number of spermatogonia is significantly reduced (Fig. 4B). These include genes involved in retinoic acid signaling that are known to impact male fertility (Livera et al. 2002). Retinoic acid receptor $\gamma$, which has been shown to control expression of Stra8 (Chiba et al. 1997), is expressed at lower levels in Taf4b-null testis (Fig. 6C). Other retinoic acid signaling molecules, including cellular retinoic acid-binding protein $(\mathrm{Crabp})$ and retinolbinding protein $(R b p)$, are reduced in Taf $4 b$-null testis as well (Fig. 6C).

Genes involved in meiosis that commences at day 9 in the mouse testes are lower in the Taf $4 b$-null testes at post-natal day 8 including Dmc1, Scp3, and Stag3 (Fig. 6D; Prieto et al. 2001; Hunt and Hassold 2002). Bard1, which has recently been identified as a pro-apoptotic factor in the testes (Feki et al. 2004), is low in day 8 Taf $4 b$ null testes. Bmp8b, which is required for gonocyte proliferation (Zhao et al. 1996), is expressed at lower levels in Taf $4 b$-null testes by RT-PCR at day 8 but not at day 0 or day 3 (Fig. 6E; data not shown). JAGGED2, the ligand for NOTCH1, is also expressed at lower levels in Taf $4 b$-null males. Mice null for Jagged2 die just after birth, precluding any study of fertility (Jiang et al. 1998). However, JAGGED2 has been functionally implicated in spermatogenesis by in vitro studies where immunodepletion of JAGGED2 or NOTCH1 causes the disappearance of post-meiotic germ cells in culture (Fig. 6E; Hayashi et al. 2001). Thus, even before puberty, several molecular abnormalities are apparent in Taf $4 b$-null testes.

\section{Discussion}

Spermatogenesis is a complex process that involves specialized transcriptional activity (Sassone-Corsi 2002). Here we show that TAF4b, a cell-type-specific component of the TFIID complex involved in RNA polymerase II transcription, is essential for continuous spermatogenesis and a requisite for fertility in mice. Although males null for $T a f 4 b$ are able to sire one litter, they become infertile. The studies described herein were conducted in a pure $\mathrm{C} 57 \mathrm{BL} / 6$ background, and in this background penetrance is $100 \%$. The initial characterization of Taf $4 b$ null mice was conducted in a mixed genetic background in which some of the males were fertile (Freiman et al. 2001). The Taf $4 b$-null phenotype with the pure C57BL/6 background is characterized specifically by decreases in germ cell (gonocyte) number as early as day 2, lack of spermatogonial proliferation leading to progressive decreases in testes size, eventual depletion of sper- 


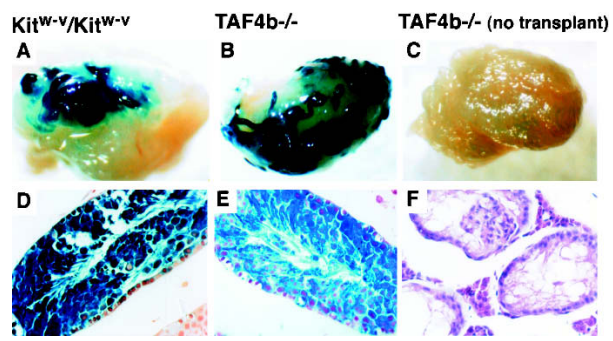

Figure 5. Germ cell transplantation surgeries were performed using germ cell-deficient $\mathrm{Kit}^{W-V} / \mathrm{Kit}^{W}(A)$ or Taf $4 b$-null $(B)$ males. $(C)$ Uninjected Taf $4 b$-null testes are also shown. $(A-C)$ Recipient males were sacrificed 3 mo after transplantation, and their testes were stained $\beta$-galactosidase to detect donor cells. Successful colonization (blue-stained tubules) were seen in $6 / 8$ $\mathrm{Kit}^{W-V} / \mathrm{Kit}^{W}$ and 6/7 Taf $4 \mathrm{~b}$-null testes transplanted. $(D-F)$ Spermatogenesis was also observed in the colonized tubules but not in the nontransplanted testes.

matogonia, and defects in spermiogenesis clearly evident by $8 \mathrm{wk}$.

Successful maintenance of spermatogenesis requires the establishment of a pool of spermatogonial stem cells that self-renew and differentiate into mature sperm. In the newborn testes, gonocytes are located at the center of the seminiferous tubule. The initial colonization of the gonad by germ cells appears to be normal since at birth Taf $4 b$-null males have comparable numbers of gonocytes per seminiferous tubule relative to wild type and heterozygous littermates. Thus, factors that regulate germ cell migration and early stages of proliferation appear intact (McLaren 2003). Shortly after birth gonocytes migrate to the basement membrane, differentiate into spermatogonial stem cells, and proliferate /de Rooij and Russell 2000), a process that leads to the appearance of functional spermatogonial stem cells at post-natal day 3-4 (McLean et al. 2003). These spermatogonial stem cells proliferate rapidly prior to puberty resulting in a 39 -fold increase in the number of germ cell stem cells between birth and adulthood (Shinohara et al. 2001). The observed deficiency in germ cells in Taf4b-null males beginning at day 2-3 corresponds with this critical stage of stem cell development and expansion, suggesting that the inability of adult Taf $4 b$-null males to maintain spermatogenesis might be due to defects in spermatogonial stem cell function. This hypothesis is further substantiated by the ability of transplanted wild-type germ cell stem cells to colonize in seminiferous tubules of adult germ cell-depleted Taf $4 b$-null mice.

The phenotype observed in Taf $4 b$-null males differs from other previously described mouse models of male infertility. Other knockout mice that exhibit germ cell deficiency just after birth including Pin1-null and $B m p 8 b$-null mice exhibit defects in primordial germ cell proliferation prior to birth (Zhao et al. 1996; Atchison and Means 2003; Atchison et al. 2003). Since the Taf4bnull mice can complete spermatogenesis and spermiogenesis leading to the production of functional sperm and healthy offspring, the genes that mediate these events also seem to be largely unaffected at least in young males. Increased apoptosis also does not appear to underlie the $T a f 4 b$-null phenotype, which is in contrast to many other mouse models of infertility (Matzuk and Lamb 2002). The endocrine system also appears to be largely intact since serum levels of the pituitary gonadotropins and testosterone are normal in young Taf $4 b$-null mice. Although FSH levels increase in older mice, this could be a consequence of the altered function of the tubules with germ cell loss rather than a cause of the abnormal phenotype. The defects in spermiogenesis observed at $12 \mathrm{wk}$ of age, but not at $8 \mathrm{wk}$ of age, are most likely secondary to the degeneration of the testes that is characterized by loss of germ cells and elevated FSH levels.

The GDNF signaling pathway, which is regulated by FSH, has been shown to be critical for germ cell stem cell fate decision (Meng et al. 2000; Tadokoro et al. 2002). Mice with decreased Gdnf levels are fertile; however, spermatogonial stem cells differentiate at inappropriately high levels resulting in seminiferous tubules depleted of sperm in older animals. Conversely, mice overexpressing Gdnf are infertile because germ cell stem cells proliferate but do not differentiate (Meng et al. 2000). GDNF, which is secreted by Sertoli cells, signals through the receptor GFR1 $\alpha$, which activates the recep-
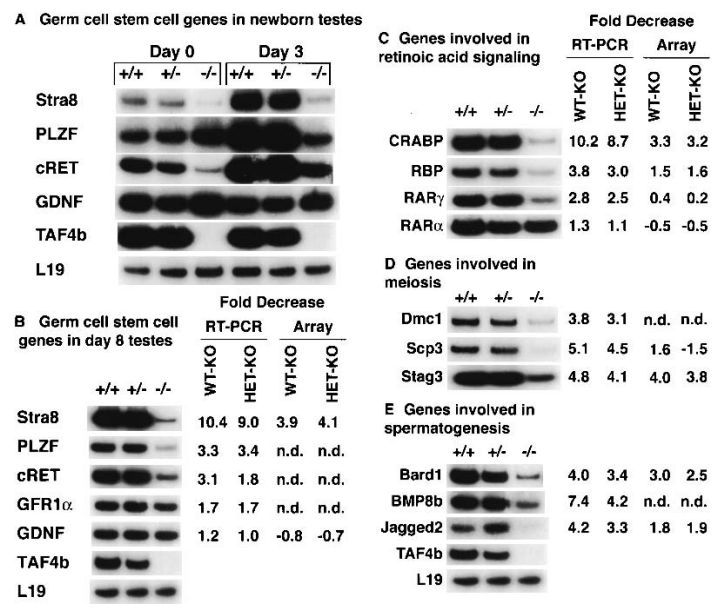

Figure 6. (A) Semiquantitative RT-PCR was performed using RNA isolated from day 0 and day 3 wild-type and $T a f 4 b$ heterozygous and null testes using specific primer pairs against Stra8, Plzf, c-Ret, and Gdnf. For each sample at least five animals were pooled. Microarray analysis was also performed using day 8 testes. RT-PCR reactions were done two to three times. Representative autoradiographs are shown. $(B-E)$ Semiquantitative RT-PCR was performed using RNA isolated from day 8 wild-type and Taf $4 b$ heterozygous and null testes. Statistical analyses were based on the results of RT-PCR with six individual samples for each group. All values are normalized to the internal control L19. Fold change was calculated comparing knockout to wild-type (left column) or heterozygous (right column) values. Representative autoradiographs are included. Statistical significance was calculated by two-way ANOVA. For $G d n f$ there is not a statistically significant difference. For Rar $\alpha$ and $G f r 1 \alpha, p<0.05$; for all other genes assayed, $p<0.01$. Genes that were not included on the array are designated as "n.d." 
tor tyrosine kinase c-Ret (Sariola and Saarma 2003; Kubota et al. 2004). Transgenic mice expressing a dominant-negative $c$-Ret mutation have normal testes on the day of birth. However, by post-natal day 10, mutant $c$-Ret mice have fewer germ cells, a phenotype similar to that observed in Taf4b-null males (Jain et al. 2004). The observed deficiency of $c$-Ret in Taf $4 b$-null males coupled with the evidence that GDNF signaling is important to germ cell stem cell function directly implicates a role for TAF4b in these germ cell stem cells. Since $c$-Ret is deficient in Taf $4 b$-null testes as early as the day of birth, when Taf $4 b$-null males have comparable numbers of gonocytes relative to wild-type males of the same age, $c$-Ret could be a direct target of TAF4b.

PLZF (promyelocytic leukemia-associated protein), a zinc finger transcription factor involved in germ cell stem cell self-renewal (Buaas et al. 2004), is expressed at normal levels in Taf $4 b$-null testes at day 0 , but at lower levels in Taf $4 b$-null testes at day 3. This deficiency of Plzf at day 3 is probably the result of the presence of fewer germ cell stem cells at this time in Taf $4 b$-null testes. Thus, Plzf is most likely not a direct target of $T a f 4 b$; however, decreased expression of this germ cell stem cell marker in Taf $4 b$-null males at day 3 provides further evidence that loss of TAF4B may cause germ cell stem cell deficiency due to impaired proliferation.

The role of retinoic acid signaling in male fertility is well established (Livera et al. 2002). Stra8, a RAR $\gamma$-responsive gene, (Chiba et al. 1997) is the most dramatically down-regulated gene in Taf $4 b$-null testes by semiquantitative RT-PCR. Stra8 is deficient as early as the day of birth, when Taf $4 b$-null testes have comparable numbers of germ cells compared to wild type and heterozygous littermates. Although the function of STRA8 is not yet known, STRA8 has been shown to be expressed in premeiotic spermatogonia and embryonic oocytes and has been used as a marker of spermatogonial stem cells (Oulad-Abdelghani et al. 1996; Menke et al. 2003; Lassalle et al. 2004). These results suggest that the infertility in Taf $4 b$-null mice could also be caused in part by depletion of Stra8. Whether or not TAF4b is targeted to the promoter of RAR $\gamma$ or Stra8 remains to be determined.

Control of spermatogenesis requires specialized transcriptional machinery in multiple species including Drosophila, Xenopus, mouse, and human (Martianov et al. 2001; Zhang et al. 2001; Wang and Page 2002; Han et al. 2003; Hiller et al. 2004). These alternative general transcription factors may be direct targets of testis-specific factors or function in the initiation of expression-specific genes in the testis that are expressed from alternative promoters (Sassone-Corsi 2002). The observation that a specific TBP-associated factor, TAF4b, appears to be required for the expression of several genes thought to regulate spermatogonial stem cell proliferation provides evidence that the complex events controlling germ cell proliferation in the neonatal mouse testes between postnatal day 0 and day 3 unexpectedly require a specialized component of the preinitiation complex (Hochheimer and Tjian 2003). One would predict that TAF4b, like the other TAFs, is an essential coactivator for specific transcription factors that impact chromatin structure and gene transcription (Veenstra and Wolffe 2001). Future studies at the molecular level will investigate what chromatin-directed events occur in spermatogonial stem cells and what specific functions TAF4b plays in these molecular events.

In summary, mice null for Taf $4 b$ exhibit a unique testicular phenotype that includes normal fertility at early ages followed by a complete loss of fertility by $12 \mathrm{wk}$ of age, characterized spermiogenesis defects, loss of germ cells, and testicular degeneration. Although other components of the RNA Polymerase II basal transcription apparatus impact meiosis (Zhang et al. 2001; Hiller et al. 2004), TAF4b is required for mitosis specifically in gonocytes and spermatogonia. TAF4b appears to be essential for germ cell stem cell differentiation and proliferation, which are required for the maintenance of fertility in adult males. Thus, TAF4b, a component of the TFIID complex of RNA polymerase II basal transcription machinery, is essential for the maintenance of spermatogenesis, most specifically the proliferation of spermatogonial stem cells.

\section{Materials and methods}

\section{Animals}

Mice null for $T a f 4 b$ were generated by an insertion of a neomycin-resistance cassette in the reverse orientation into the sixth exon of the Taf $4 b$ gene as previously described (Freiman et al. 2001). Heterozygous mice were backcrossed to the inbred C57BL/6 strain (Harlan Sprauge Dawley, Inc.). Mice were maintained on a 14L:10D cycle with free access to food and water in the vivarium at Baylor College of Medicine. For natural breeding experiments, males were housed with one or two females. Neonatal males were collected from timed pregnancies. Tissues were fixed in Bouin's fixative or frozen for RNA isolation. Mice were maintained in accordance with the NIH Guide for the Care and Use of Laboratory Animals with institutional oversight by Baylor College of Medicine.

\section{Fertilization experiments}

Males participating in mating experiments were housed in separate cages for the duration. Immature female C57BL/6 (d25) were obtained from Harlan Sprauge Dawley, Inc. or from heterozygous crossings and injected with 5 IU of pregnant mare serum gonadotropin (PMSG, Gestyl) purchased from the Professional Compounding Center of America to stimulate follicle growth, followed $48 \mathrm{~h}$ later with $5 \mathrm{IU}$ of human chorionic gonadotropin (hCG; Pregnyl) from Organon Special Chemicals to stimulate ovulation and luteinization. Following treatment with hCG, females were placed in cages with a single male. After $24 \mathrm{~h}$ vaginal plugs were observed, and after $52 \mathrm{~h}$ females were sacrificed and oviducts were collected. Oocytes were collected from the oviduct, counted, and examined by light microscopy for fertilization stage.

\section{Germ cell transplantation}

Cells for transplantation were isolated from the testes of the transgenic mouse line ROSA26 (originally from The Jackson 
Laboratory), which are maintained on a C57BL/6 $\times 129 / \mathrm{Sv}$ genetic background and express the Escherichia coli LacZ gene. Many cell types, including germ cells of all stages of differentiation, stain positively for $\beta$-galactosidase ( $\beta$-gal), a characteristic that allows accurate tracking of the transplanted cells. The male mice were surgically made cryptorchid at age 6-8 wk to minimize the number of haploid germ cells in the donor cell population. On average, six to eight donor mice were killed and their testes were harvested 6-8 wk post-operatively. A singlecell suspension was prepared using a two-step enzymatic digestion procedure as described previously (Ogawa et al. 1997). The cells were resuspended in medium containing DMEM, 5\% HEPES, and $10 \%$ fetal bovine serum.

Transplantation surgeries were performed using 4-6-mo-old $\mathrm{Kit}^{W-v} / \mathrm{Kit}^{W}$ (The Jackson Laboratory) or Taf $4 \mathrm{~b}$-null testes. Mice were killed 3 mo after transplantation. The testes were immediately fixed in fresh $\mathrm{CHO}^{\prime}$ s fixative $3 \%$ paraformaldehyde, $0.2 \%$ glutaraldehyde, and $2 \%$ sucrose in $\mathrm{PBS}$ at $\mathrm{pH} 7.5$ ) at $4^{\circ} \mathrm{C}$ for $2 \mathrm{~h}$. Whole-mount colorimetric $\beta$-gal staining was performed using the modified protocols from Specialty Media. Because it is difficult to stain the tissue evenly with this method, we bivalved the testes for optimal staining. After rinsing with PBS and washing with buffer solutions, the testes were incubated in COMPLETE $\beta$-Gal Tissue Stain Solution (Specialty Media) for 3 $\mathrm{h}$ at $37^{\circ} \mathrm{C}$. Positive colonization with donor cells is apparent from the blue color generated with this assay. The testes were then refixed with CHO's fixative for $12 \mathrm{~h}$ (or overnight). Histology sections were obtained as described below and counterstained with nuclear fast red.

\section{Histology and immunohistochemistry}

Testes were collected and fixed in Bouin's fixative overnight, dehydrated in $70 \%$ ethanol, and embedded in paraffin. Sectioned tissues were dehydrated and stained with hematoxylin and eosin then re-dehydrated and mounted. The cellular and subcellular localization of germ cell nuclear antigen and phospho-histone H3 were analyzed by immunostaining Bouin'sfixed and paraffin-embedded testes. Sections $(7 \mu \mathrm{M})$ were rehydrated and boiled in $0.1 \%$ hydrogen peroxide followed by PBS washes. Sections were then incubated with $20 \%$ nonimmune goat serum to block nonspecific sites, followed by incubation with the 10D9G11 (GCNA1) monoclonal antibody at $33^{\circ} \mathrm{C}$ for 90 min (kindly provided by G.C. Enders, University of Kansas, Kansas City, Kansas) or with a polyclonal antibody against Phospho-histone $\mathrm{H} 3$ (1:60) overnight at $4^{\circ} \mathrm{C}$ (Cell Signaling Technology). After washing in PBS, horseradish peroxidase-conjugated anti-Rat IgM $\mu$ diluted to a concentration of 1:450 was applied for $60 \mathrm{~min}$ at room temperature (Pierce Biotechnology, Inc.). Sections were incubated with DAB substrate (3,3'-diaminobenzidine) (Vector Laboratories) for $1 \mathrm{~min}$, dehydrated, counterstained with hemotoxylin and mounted. The number of positive cells per seminiferous tubule was counted for at least six individual animals and averaged. At least 60 seminiferous tubules were counted per testis. One-way ANOVA was used for statistical analysis.

\section{Western blot}

Total protein extracts were prepared from testes of adult $T a f 4 b$ heterozygous and null mice. Testes were dounce homogenized in extraction buffer (100 mM HEPES at $\mathrm{pH} 7.6,400 \mathrm{mM} \mathrm{NaCl}$, $1.5 \mathrm{mM} \mathrm{MgCl} 2,0.1 \%$ NP-40, 10\% glycerol, 0.5× COMPLETE [Roche]), $1 \mathrm{mM}$ PMSF, $20 \mu \mathrm{M}$ leupeptin, $20 \mathrm{KIU}$ of aprotinin, 10 $\mathrm{mM} \mathrm{NaF}, 5 \mathrm{mM} \mathrm{Na}_{3} \mathrm{VO}_{4}$, and $2 \mathrm{mM} \mathrm{Na}_{4} \mathrm{P}_{2} \mathrm{O}_{7}$ ), incubated on ice for $20 \mathrm{~min}$, and centrifuged. A total of $30 \mu \mathrm{g}$ of protein was separated on a $7 \%$ SDS-polyacrylamide gel. Western blot analy- sis was performed with anti-TAF4b rabbit polyclonal antisera diluted 1:2500, goat anti-rabbit IgG-horseradish peroxidase (Pierce) diluted 1:13,000 and visualized with SuperSignal Chemiluminscent Substrate (Pierce).

\section{TUNEL assay}

TUNEL assays were performed on Bouin's fixed paraffin embedded sections according to the manufacturer's instructions using the ApopTag Plus Peroxidase In Situ Apoptosis Detection Kit (Serologicals Corporation).

\section{Sperm assays}

Epidydimides were dissected and sheared in Modified BWW Medium (Irvine Scientific) and incubated for $30 \mathrm{~min}$. For sperm motility counts, live sperm were immediately spread onto a slide, viewed, and scored visually as motile or immotile. For whole sperm counts, sperm was immobilized by treatment with water and put into a hemocytometer for counting. For acrosome reactions, sperm were spread onto slides and allowed to dry overnight. On the following morning, sperm were stained with Rhodamine-labeled Pisum Sativum Agglutinin for $10 \mathrm{~min}$ (Vector Laboratories), washed, and mounted with $50 \%$ glycerol. Positive-staining acrosomes were visualized and scored using a fluorescent microscope.

\section{Hormone assays}

Blood was drawn by cardiac puncture. Serum was separated by centrifugation ( $2 \mathrm{~min}, 16,000 \mathrm{rpm}$ ) in microtainer brand tubes (Becton Dickinson), frozen and stored at $-80^{\circ} \mathrm{C}$. Radioimmunoassays for FSH, $\mathrm{LH}$, and $\mathrm{T}$ were performed by the Ligand Assay and Analysis Core (University of Virginia) as previously described (Gay et al. 1970; Fallest et al. 1995) or by using the commercially available testosterone RIA kit from Diagnostic Systems Laboratories, Inc. or Diagnostic Products Corporation.

\section{RNA isolation}

Whole testis RNA was obtained by tissue homogenization in TRIzol reagent (Line Technologies, Inc.), followed by RNA precipitation in isopropanol. Recovered RNA was then resuspended in DEPC-treated water and verified as DNA free by PCR. RNA was quantitated and stored at $-80^{\circ} \mathrm{C}$ until use.

\section{Microarray}

Total RNA was purified from day 8 testes of Taf $4 b$ wild-type, heterozygous, and null littermates. For cDNA synthesis, $12 \mu \mathrm{g}$ of total RNA was used in a reverse transcription reaction at $42^{\circ} \mathrm{C}$ using a $\mathrm{T} 7-(\mathrm{dT})_{24}$ primer and superscript II reverse transcriptase (Invitrogen). In vitro transcribed cRNA was prepared using half of the cDNA sample, and $15 \mu \mathrm{g}$ of fragmented cRNA was hybridized to Affymetrix U74Av2 Murine Genome highdensity oligonucleotide arrays as per the manufacturer's protocols (Affymetrix). Data analysis for individual arrays and single comparisons was performed using Microarray Suite 5.0, and cross-comparison analysis of replicate experiments was performed using Data Mining Tool 2.0 (Affymetrix). Microarray analyses were repeated with a second group of animals to verify accuracy.

\section{$R T-P C R$}

Total RNA (300 ng) was reverse-transcribed using poly/dT) (Amersham Pharmacia Biotech) and avian myeloblastosis virus-re- 
verse transcriptase (Promega Corp.) at $42^{\circ} \mathrm{C}$ for $75 \mathrm{~min}$ and $95^{\circ} \mathrm{C}$ from $5 \mathrm{~min}$. PCR primers were generated using a Web-based prediction algorithm (http://www.genome.wi.mit.edu/genomesoftware/other/primer3.html), and cDNA was amplified using Taq Polymerase (Promega Corp.). ${ }^{32}$ P-CTP was added to the reaction mixture for incorporation and reactions were completed in 25-30 cycles of PCR at $95^{\circ} \mathrm{C}$ for $30 \mathrm{sec}, 59^{\circ} \mathrm{C}$ for $45 \mathrm{sec}$, and $72^{\circ} \mathrm{C}$ for $60 \mathrm{sec}$. The amplified cDNA products were resolved on a $5 \%$ polyacrylamide gel, which was dried and exposed to film. The radioactive PCR product bands were quantified by using a Storm 860 PhosphorImager (Molecular Dynamics, Inc.). To determine the linear range of amplification for specific mRNAs, $300 \mathrm{ng}$ of RNA was reverse-transcribed and amplified in a range of cycle numbers. The primer pairs and number of cycles used are listed in Supplementary Table 1. Sequences for the amplified PCR products were verified by subcloning and sequencing at the Baylor College of Medicine Sequencing Core.

\section{Statistics}

All statistics were analyzed with Prism GraphPad software. For all data sets, standard $t$-tests or one-way and two-way ANOVA were used, and standard error of means were calculated as appropriate. Statistical significance was defined as $p<0.05$.

\section{Acknowledgments}

We thank Yoh Isogai for expert advice on microarray hybridization conditions and data assembly and analysis. We are grateful to G.C. Enders (University of Kansas) for generously providing the GCNA-1 antibody. We thank Claire Lo, Lata Murthy, and Lyann Mitchell for technical assistance. We thank Karen Hirschi, Austin Cooney, Derek Boerboom, and Chad Wayne for critical review of the manuscript. This work was supported by NIH training grant HD-07165 (supporting A.E.F.); NIH Grants HD-16272 (to J.S.R.), HD-36289 (to D.J.L.), and HD-29428 (to P.L.M.); ACS grant PF-02-113-01-GMC (to K.G.G.); American Foundation for Urologic Disease grants (to K.C.L. and D.J.L.); and NCI Grant CA-25417 (to R.T). The University of Virginia Ligand Assay core was supported by the NICHD/NIH through cooperative agreement U54 HD28934 as part of the Specialized Cooperative Centers Program in Reproductive Research.

\section{References}

Atchison, F.W. and Means, A.R. 2003. Spermatogonial depletion in adult Pin1-deficient mice. Biol. Reprod. 69: 19891997.

Atchison, F.W., Capel, B., and Means, A.R. 2003. Pin1 regulates the timing of mammalian primordial germ cell proliferation. Development 130: 3579-3586.

Buaas, F.W., Kirsh, A.L., Sharma, M., McLean, D.J., Morris, J.L., Griswold, M.D., de Rooij, D.G., and Braun, R.E. 2004. Plzf is required in adult male germ cells for stem cell self-renewal. Nat. Genet. 36: 647-652.

Chiba, H., Clifford, J., Metzger, D., and Chambon, P. 1997. Distinct retinoid $\mathrm{X}$ receptor-retinoic acid receptor heterodimers are differentially involved in the control of expression of retinoid target genes in F9 embryonal carcinoma cells. Mol. Cell. Biol. 17: 3013-3020.

de Rooij, D.G. and Russell, L.D. 2000. All you wanted to know about spermatogonia but were afraid to ask. I. Androl. 21: 776-798.

Enders, G.C. and May II, J.J. 1994. Developmentally regulated expression of a mouse germ cell nuclear antigen examined from embryonic day 11 to adult in male and female mice. Dev. Biol. 163: 331-340.

Fallest, P.C., Trader, G.L., Darrow, J.M., and Shupnik, M.A. 1995. Regulation of rat luteinizing hormone beta gene expression in transgenic mice by steroids and a gonadotropinreleasing hormone antagonist. Biol. Reprod. 53: 103-109.

Feki, A., Jefford, C.E., Durand, P., Harb, J., Lukas, H., Krause, K.H., and Irminger-Finger, I. 2004. BARD1 expression during spermatogenesis is associated with apoptosis and hormonally regulated. Biol. Reprod. 71: 1614-1624.

Freiman, R.N., Albright, S.R., Zheng, S., Sha, W.C., Hammer, R.E., and Tjian, R. 2001. Requirement of tissue-selective TBP-associated factor TAF 105 in ovarian development. Science 293: 2084-2087.

Gay, V.L., Midgley Jr., A.R., and Niswender, G.D. 1970. Patterns of gonadotrophin secretion associated with ovulation. Fed. Proc. 29: 1880-1887.

Giuili, G., Tomljenovic, A., Labrecque, N., Oulad-Abdelghani, M., Rassoulzadegan, M., and Cuzin, F. 2002. Murine spermatogonial stem cells: Targeted transgene expression and purification in an active state. EMBO Rep. 3: 753-759.

Han, S., Xie, W., Hammes, S.R., and DeJong, J. 2003. Expression of the germ cell-specific transcription factor ALF in Xenopus oocytes compensates for translational inactivation of the somatic factor TFIIA. J. Biol. Chem. 278: 45586-45593.

Hayashi, T., Kageyama, Y., Ishizaka, K., Xia, G., Kihara, K., and Oshima, H. 2001. Requirement of Notch 1 and its ligand jagged 2 expressions for spermatogenesis in rat and human testes. J. Androl. 22: 999-1011.

Hiller, M., Chen, X., Pringle, M.J., Suchorolski, M., Sancak, Y., Viswanathan, S., Bolival, B., Lin, T.Y., Marino, S., and Fuller, M.T. 2004. Testis-specific TAF homologs collaborate to control a tissue-specific transcription program. Development 131: 5297-5308.

Hochheimer, A. and Tjian, R. 2003. Diversified transcription initiation complexes expand promoter selectivity and tissuespecific gene expression. Genes \& Dev. 17: 1309-1320.

Hunt, P.A. and Hassold, T.J. 2002. Sex matters in meiosis. Science 296: 2181-2183.

Jain, S., Naughton, C.K., Yang, M., Strickland, A., Vij, K., Encinas, M., Golden, J., Gupta, A., Heuckeroth, R., Johnson Jr., E.M., et al. 2004. Mice expressing a dominant-negative Ret mutation phenocopy human Hirschsprung disease and delineate a direct role of Ret in spermatogenesis. Development 131: 5503-5513.

Jiang, R., Lan, Y., Chapman, H.D., Shawber, C., Norton, C.R., Serreze, D.V., Weinmaster, G., and Gridley, T. 1998. Defects in limb, craniofacial, and thymic development in Jagged2 mutant mice. Genes \& Dev. 12: 1046-1057.

Kubota, H., Avarbock, M.R., and Brinster, R.L. 2004. Growth factors essential for self-renewal and expansion of mouse spermatogonial stem cells. Proc. Natl. Acad. Sci. 101: 16489-16494.

Lassalle, B., Bastos, H., Louis, J.P., Riou, L., Testart, J., Dutrillaux, B., Fouchet, P., and Allemand, I. 2004. 'Side Population' cells in adult mouse testis express Bcrp1 gene and are enriched in spermatogonia and germinal stem cells. Development 131: 479-487.

Livera, G., Rouiller-Fabre, V., Pairault, C., Levacher, C., and Habert, R. 2002. Regulation and perturbation of testicular functions by vitamin A. Reproduction 124: 173-180.

Martianov, I., Fimia, G.M., Dierich, A., Parvinen, M., SassoneCorsi, P., and Davidson, I. 2001. Late arrest of spermiogenesis and germ cell apoptosis in mice lacking the TBP-like TLF/TRF2 gene. Mol. Cell 7: 509-515. 
Martianov, I., Brancorsini, S., Gansmuller, A., Parvinen, M., Davidson, I., and Sassone-Corsi, P. 2002. Distinct functions of TBP and TLF/TRF2 during spermatogenesis: Requirement of TLF for heterochromatic chromocenter formation in haploid round spermatids. Development 129: 945-955.

Matzuk, M.M. and Lamb, D.J. 2002. Genetic dissection of mammalian fertility pathways. Nat. Cell Biol. 4 Suppl: s41-s49.

McLaren, A. 2003. Primordial germ cells in the mouse. Dev. Biol. 262: 1-15.

McLean, D.J., Friel, P.J., Johnston, D.S., and Griswold, M.D. 2003. Characterization of spermatogonial stem cell maturation and differentiation in neonatal mice. Biol. Reprod. 69: 2085-2091.

Meng, X., Lindahl, M., Hyvonen, M.E., Parvinen, M., de Rooij, D.G., Hess, M.W., Raatikainen-Ahokas, A., Sainio, K., Rauvala, H., Lakso, M., et al. 2000. Regulation of cell fate decision of undifferentiated spermatogonia by Gdnf. Science 287: 1489-1493.

Menke, D.B., Koubova, J., and Page, D.C. 2003. Sexual differentiation of germ cells in XX mouse gonads occurs in an anterior-to-posterior wave. Dev. Biol. 262: 303-312.

Ogawa, T., Arechaga, J.M., Avarbock, M.R., and Brinster, R.L. 1997. Transplantation of testis germinal cells into mouse seminiferous tubules. Int. J. Dev. Biol. 41: 111-122.

Oulad-Abdelghani, M., Bouillet, P., Decimo, D., Gansmuller, A., Heyberger, S., Dolle, P., Bronner, S., Lutz, Y., and Chambon, P. 1996. Characterization of a premeiotic germ cellspecific cytoplasmic protein encoded by Stra8, a novel retinoic acid-responsive gene. J. Cell Biol. 135: 469-477.

Ozer, J., Moore, P.A., and Lieberman, P.M. 2000. A testis-specific transcription factor IIA (TFIIA $\tau$ ) stimulates TATA-binding protein-DNA binding and transcription activation. J. Biol. Chem. 275: 122-128.

Pointud, J.C., Mengus, G., Brancorsini, S., Monaco, L., Parvinen, M., Sassone-Corsi, P., and Davidson, I. 2003. The intracellular localisation of TAF7L, a paralogue of transcription factor TFIID subunit TAF7, is developmentally regulated during male germ-cell differentiation. J. Cell Sci. 116: 18471858.

Prieto, I., Suja, J.A., Pezzi, N., Kremer, L., Martinez, A.C., Rufas, J.S., and Barbero, J.L. 2001. Mammalian STAG3 is a cohesin specific to sister chromatid arms in meiosis I. Nat. Cell Biol. 3: 761-766.

Sariola, H. and Saarma, M. 2003. Novel functions and signalling pathways for Gdnf. J. Cell Sci. 116: 3855-3862.

Sassone-Corsi, P. 1997. Transcriptional checkpoints determining the fate of male germ cells. Cell 88: 163-166.

. 2002. Unique chromatin remodeling and transcriptional regulation in spermatogenesis. Science 296: 2176-2178.

Schmidt, E.E. and Schibler, U. 1995. High accumulation of components of the RNA polymerase II transcription machinery in rodent spermatids. Development 121: 2373-2383.

Shinohara, T., Orwig, K.E., Avarbock, M.R., and Brinster, R.L. 2001. Remodeling of the postnatal mouse testis is accompanied by dramatic changes in stem cell number and niche accessibility. Proc. Nat1. Acad. Sci. 98: 6186-6191.

Tadokoro, Y., Yomogida, K., Ohta, H., Tohda, A., and Nishimune, Y. 2002. Homeostatic regulation of germinal stem cell proliferation by the Gdnf/FSH pathway. Mech. Dev. 113: 29-39.

Veenstra, G.J. and Wolffe, A.P. 2001. Gene-selective developmental roles of general transcription factors. Trends Biochem. Sci. 26: 665-671.

Verrijzer, C.P. and Tjian, R. 1996. TAFs mediate transcriptional activation and promoter selectivity. Trends Biochem. Sci. 21: 338-342.
Wang, P.J. and Page, D.C. 2002. Functional substitution for $\mathrm{TAF}_{\mathrm{II}} 250$ by a retroposed homolog that is expressed in human spermatogenesis. Hum. Mol. Genet. 11: 2341-2346.

Zhang, D., Penttila, T.L., Morris, P.L., Teichmann, M., and Roeder, R.G. 2001. Spermiogenesis deficiency in mice lacking the Trf2 gene. Science 292: 1153-1155.

Zhao, G.Q., Deng, K., Labosky, P.A., Liaw, L., and Hogan, B.L. 1996. The gene encoding bone morphogenetic protein $8 \mathrm{~B}$ is required for the initiation and maintenance of spermatogenesis in the mouse. Genes \& Dev. 10: 1657-1669. 


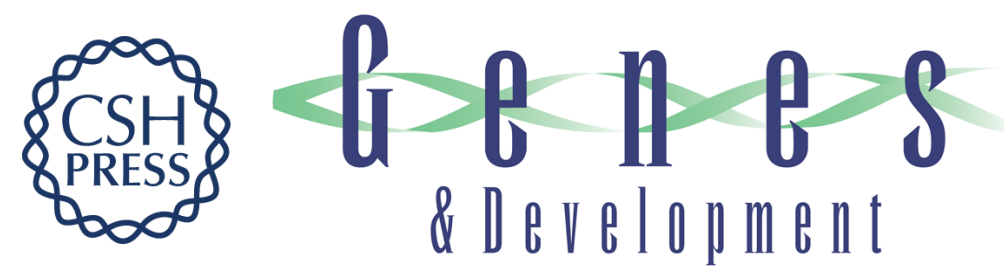

\section{Maintenance of spermatogenesis requires TAF4b, a gonad-specific subunit of TFIID}

Allison E. Falender, Richard N. Freiman, Kenneth G. Geles, et al.

Genes Dev. 2005, 19:

Access the most recent version at doi:10.1101/gad.1290105

\section{Supplemental http://genesdev.cshlp.org/content/suppl/2005/03/16/gad.1290105.DC1 Material}

References This article cites 42 articles, 22 of which can be accessed free at: http://genesdev.cshlp.org/content/19/7/794.full.html\#ref-list-1

\section{License}

Email Alerting

Receive free email alerts when new articles cite this article - sign up in the box at the top Service 